\title{
Robust Optimal Design of TMD and TLD Parameters in Reducing the Seismic Response of a Two DOF Shear Structure
}

\author{
Lovely Son ${ }^{a^{*}}$, Mulyadi Burb, and Meifal Ruslic \\ a,b,c Department of Mechanical Engineering, Andalas University, Padang, Indonesia
}

\begin{abstract}
This research proposes a technique for reducing the seismic response of a vibration system using TMD and TLD absorbers. Application of the proposed method to the dynamic model of a two DOF shear structure is evaluated. The equation of motion of the system is calculated by considering the nonlinear behavior of the fluid motion inside the TLD container. The nonlinear characteristic of the TLD absorber is modeled using a nonlinear stiffness and dashpot. Robust optimum design of the absorber parameters using Genetic Algorithm (GA) is conducted base on the mean value and the variance of the performance function, which calculated from the ratio between the system response and its excitation signal. The simulation results shown that a combination of TMD and TLD absorbers can effectively reduce the seismic response of a two DOF vibration systems.
\end{abstract}

Keywords: Vibration, Absorber, TMD, TLD, GA.

\section{INTRODUCTION}

Vibration attenuation of a large structure induced by the dynamic load has received much attention from many researchers around the world during last decades. Several methods have been proposed and the theories are developed for solving these problems. Vibration isolators using flexible mounting elements are commonly used to reduce vibration transmission from the foundation to the main structure ${ }^{1}$. Seismic retrofitting of existing structures to make them more resistant to seismic activity, ground motion, or soil failure due to earthquakes have been used by some researchers to reduce the structural response during earthquake excitation ${ }^{2}$.

The passive dynamic vibration absorbers (DVAs) are one of the several popular techniques recently used for reducing seismic response of the large structures such as high rise buildings ${ }^{3}$, bridges ${ }^{4}$ and wind turbine towers ${ }^{5}$. In comparison to the other vibration control techniques, the passive DVA has lower construction and maintenance cost. The passive DVA performance can be as good as other vibration control methods if it's parameters are well designed ${ }^{6}$.

For a multi degree of freedom (MDOF) vibration system, a combination of some passive DVAs can reduce the response of the vibration system under the specific excitation frequency range. For these type of vibration systems, the DVA parameters can be design using several optimization techniques such as Minimax $^{7}$ and Genetic Algorithm $(\mathrm{GA})^{8}$. In the previous research, the authors have evaluated the TMD and TLCD absorbers performance for reducing the vibration response of a two DOF shear structure model ${ }^{9}$. In this research, the combination of TMD and TLD absorbers are proposed to attenuate the vibration response of two DOF shear structure under the seismic load excitation.

*lovelyson@ft.unand.ac.id

\section{THEORY}

Figure 1 shows a two DOF shear structure with the TMD and TLD absorbers. Both absorbers are located at the second floor of the structure. An analogy of tuned mass damper system with nonlinear elements ${ }^{10}$ is used to model the sloshing motion of the water inside the TLD container. Therefore, the TLD system can be assumed as a one DOF vibration system with the nonlinear spring $\left(k_{w}\right)$ and dashpot $\left(c_{w}\right)$ elements as shown in Fig.1.

The structure is excited on its supporting base by a displacement function $x_{0}$. The equivalent stiffness and damping elements between the $1^{\text {st }}$ and the $2^{\text {nd }}$ floor and between the ground and the $1^{\text {st }}$ floor are denoted by $k_{e 1}, c_{e 1}$ and $k_{e 2}, c_{e 2}$, respectively.

According to the model proposed by $\mathrm{Yu}$ et al. ${ }^{10}$, the values of the nonlinear stiffness and damping elements $\left(k_{w}\right.$ and $\left.c_{w}\right)$ depend on the water container displacement amplitude $(A)$. The relation between the TLD frequency ratio $(\xi)$ and the amplitude of the water container can be expressed by:

$$
\begin{aligned}
& \xi=1.038(A / L)^{0.0034} \text { for } A / L \leq 0.03 \\
& \xi=1.59(A / L)^{0.125} \text { for } A / L \geq 0.03
\end{aligned}
$$

It should be noted that because the container is located at the $2^{\text {nd }}$ floor then the water container displacement amplitude is equal to the displacement amplitude of the $2^{\text {nd }}$ floor. The frequency ratio $\xi$ in Eq.(1) and (2) are defined as the ratio between the mass-spring analog system natural frequency and the natural frequency of the water motion inside the container $\xi=\frac{f_{\text {analog }}}{f_{\text {water }}}$ 


$$
\begin{aligned}
& f_{\text {analog }}=\frac{1}{2 \pi} \sqrt{\frac{k_{w}}{M_{w}}} \\
& f_{\text {water }}=\frac{1}{2 \pi} \sqrt{\frac{\pi g}{L} \tanh \left(\frac{\pi h_{0}}{L}\right)}
\end{aligned}
$$

$h_{0}$ and $L$ in Eq.(5) are the initial water level and the length of the container as shown in Figure 1. By inserting the frequencies in Eq.(4) and (5) into Eq.(3), we obtain the expression for the nonlinear stiffness as given by:

$k_{w}=\left(2 \pi f_{\text {water }}\right)^{2} \xi^{2} M_{w}$

The TLD damping factor is a function of the water container displacement amplitude as given by:

$\zeta=0.5(A / L)^{0.35}$

Eq.(7) can be used to obtain the fluid damping coefficient as follows:

$$
c_{w}=\left(2 \sqrt{k_{W} M_{w}}\right) \zeta
$$

The governing equation of a Two DOF shear structure with TMD and TLD as shown in Fig. 1 can be written by:

$\left[\begin{array}{cccc}M_{w} & 0 & 0 & 0 \\ 0 & M_{d} & 0 & 0 \\ 0 & 0 & M_{f 2} & 0 \\ 0 & 0 & 0 & M_{f 1}\end{array}\right]\left\{\begin{array}{c}\ddot{x}_{1} \\ \ddot{x}_{2} \\ \ddot{x}_{3} \\ \ddot{x}_{4}\end{array}\right\}+\left[\begin{array}{ccc}c_{w} & -c_{w} & 0 \\ -c_{w} & c_{w}+c_{d} & -c_{d} \\ 0 & -c_{d} & c_{d}+c_{e 1} \\ 0 & 0 & -c_{e 1}\end{array}\right.$$$
+\left[\begin{array}{cccc}
k_{w} & -k_{w} & 0 & 0 \\
-k_{w} & k_{w}+k_{d} & -k_{d} & 0 \\
0 & -k_{d} & k_{d}+k_{e} & -k_{e} \\
0 & 0 & -k_{e} & 2 k_{e}
\end{array}\right]\left\{\begin{array}{l}
x_{1} \\
x_{2} \\
x_{3} \\
x_{4}
\end{array}\right\}=\left\{\begin{array}{c}
0 \\
0 \\
0 \\
k_{e}
\end{array}\right\} x_{0}
$$

or

$$
[\mathbf{M}]\{\ddot{\mathbf{x}}\}+[\mathbf{C}]\{\dot{\mathbf{x}}\}+[\mathbf{K}]\{\mathbf{x}\}=\{\mathbf{f}\}
$$

The equation of motion in Eq.(9) is a nonlinear differential equation because the fluid parameters such as $k_{w}$ and $c_{w}$ depend on the amplitude of the displacement response as given in Eq.(6) and (8).

\section{OPTIMIZATION}

The optimization process is conducted using the Genetic Algorithm procedure $(\mathrm{GA})^{8}$. GA has been extensively used to solve of the optimization problems and has shown its superiority in obtaining nearly global optimum solution of the complex system $^{11}$. Suppose that $\boldsymbol{y}=f(\mathbf{s}, \mathbf{x})$ denotes the vector of responses for a particular set of factors, where $\mathbf{s}$ and $\mathbf{x}$ denote the signal and control factors, respectively.

The robust optimization is conducted by minimizing both the mean value and the variance of the performance function:

$\mu_{f}=E[f(\mathbf{s}, \mathbf{x})]$

$\sigma_{f}^{2}=E\left[\left(f(\mathbf{s}, \mathbf{x})-\mu_{f}(\mathbf{s}, \mathbf{x})\right)^{2}\right]_{(12)}$

For a two DOF shear structure with TMD and TLD as shown in Fig.1, the performance function is calculated based on the frequency response function, which indicates the ratio between the system response and its excitation signal as given by: $\alpha_{j k}\left(\omega, m_{r}, c_{r}, k_{r}\right)=\sum_{r=1}^{N} \frac{\left(\Phi_{j r}\right)\left(\Phi_{k r}\right)}{\left(k_{r}-\omega^{2} m_{r}\right)+i\left(\omega c_{r}\right)}$

Where $\boldsymbol{\Phi}, \mathbf{m}_{\mathbf{r}}, \mathbf{c}_{\mathbf{r}}$ and $\mathbf{k}_{\mathbf{r}}$ are the eigenvector, modal mass, modal damping and modal stiffness of the system, respectively. Meanwhile, $N$ is the degree of freedom of the structures with the absorbers.

\section{RESULTS AND DISCUSSION}

The simulation parameters of a Two DOF shear structure and the absorbers are listed in Table 1.

To conduct the optimization process, the maximum displacement of the TLD water container is allowed to undergo variation $0.01<A / L<0.06$ and the excitation frequency range from 0 until $10 \mathrm{~Hz}$ is considered. The performance function $f$ is defined as the response function measured at $m_{f 2}$ for a given excitation at $m_{f 1}$, therefore

$$
f=\alpha_{34}\left(\omega, m_{r}, c_{r}, k_{r}\right)
$$

The mean and the standard deviation of the maximum nondimensional response of mass $m_{f 2}$ is calculated by

$$
\begin{aligned}
& \mu_{f}=E\left[\max \left(w f_{1}\right)+\max \left((1-w) f_{2}\right)\right] \\
& \sigma_{f}^{\theta}=E\left[\left[\left(\dot{x}_{\max }\left(w f_{1}\right)+\max \left((1-w) f_{2}\right)-\mu_{f}\right)^{2}\right]\right. \\
& 0 \\
& \text { Where } \\
& f_{1}^{C} f \mid \operatorname{at}^{c_{2}} \dot{x}_{L_{1}}<\omega<\omega_{R_{1}} \\
& c_{e l f}+\underline{c}_{2 f} f \dot{a t} \dot{\omega}_{L_{2}}<\omega<\omega_{R_{2}}
\end{aligned}
$$

Variables $w_{L 1}$ and $w_{R 1}$ denote the left and the right side boundary of the first natural frequency. The same rule is also implemented to $w_{L 2}$ and $w_{R 2}$ for the second natural frequency. The weighting factors $w \in\left[\begin{array}{ll}0 & 1\end{array}\right]$ represent the relative importance of the two frequency regions. Here the expected value is evaluated for the uncertain fluid parameters $k_{w}$ and $c_{w}$ due to variation of the maximum water container displacement $(A / L)$. The design variables for the absorber are $L, B, h_{0}, c_{d}$ and $k_{d}$. With lower and upper bounds given by $L \in\left[\begin{array}{ll}0.1 & 0.2\end{array}\right]$, $B \in\left[\begin{array}{ll}0.01 & 0.1\end{array}\right], h_{0} \in\left[\begin{array}{ll}0.001 & 0.05\end{array}\right], c_{d} \in\left[\begin{array}{ll}0.001 & 5\end{array}\right]$ and $k_{d} \in\left[\begin{array}{ll}2 & 1000\end{array}\right]$. Therefore, the robust design of TLD and TMD may be formulated as follows:

Minimize : $\left[\mu_{f}\left(L, B, h_{0}, c_{d}, k_{d}\right), \sigma_{f}\left(L, B, h_{0}, c_{d}, k_{d}\right)\right]$

Subject to : $0.01 \leq \frac{A}{L} \leq 0.06,0.1 \leq L \leq 0.2$,

$$
\begin{aligned}
& 0.01 \leq B \leq 0.1, \quad 0.001 \leq h_{0} \leq 0.05 \\
& 0.001 \leq c_{d} \leq 5 \text { and } 2 \leq k_{d} \leq 1000
\end{aligned}
$$

Since there are only two objective functions $\mu_{f}$ and $\sigma_{f}$, the two functions are combined into a single objective function $G$

$$
G=\eta \mu_{f}+(1-\eta) \sigma_{f} ; \quad \eta \in\left[\begin{array}{ll}
0 & 1
\end{array}\right]
$$

Optimization of the objective function $G$ in Eq. (19) is conducted using the Genetic Algorithm (GA) procedure ${ }^{6}$. The optimum parameters of the absorbers obtained using GA optimization are $L=0.1 \mathrm{~m}, B=0.023 \mathrm{~m}, h_{0}=0.049 \mathrm{~m}, c_{d}=$ $4.97 \mathrm{Ns} / \mathrm{m}$ and $k_{d}=138 \mathrm{~N} / \mathrm{m}$. The simulation results of the acceleration response calculated at the second floor for low and high excitation force are shown in Fig. 2 and Fig. 3. In this simulation, the harmonic excitation with the frequency range from 0 to $10 \mathrm{~Hz}$ is applied to the base of the structure.

In order to analyze the structure response under the seismic loading, the scaled model of El-Centro earthquake excitation signal is applied to the base of the structure. Comparison of the 
structure response with and without the absorber is depicted in Fig. 4 and Fig. 5 for low level and high level excitation force, respectively.

Comparison of the maximum response amplitude using several excitation levels is depicted in Fig. 6. For the low level excitation amplitude, the maximum displacement response is $0.0025 \mathrm{~m}$. Fig. 7 shows the ratio between the maximum displacement response with and without absorber. It can be shown from Fig. 7 that the variations of the displacement ratio are small. This result shows that the proposed DVA parameters design method is robust to the variation of the excitation amplitude level.

\section{Conclusion}

Vibration suppression of a Two DOF shear structure using combination of TMD and TLD absorbers has been conducted. Both absorbers are located at the second floor of the structure. The dynamic model of the system has derived and the numerical study is conducted to evaluate the absorbers' performance. The absorber parameters are optimized using the Genetic Algorithm (GA) procedure.

The optimization is implemented by minimizing both the mean value and the variance of the performance function, which calculated from the ratio between the structure response and the excitation signal. The simulation results show that the absorbers are effective for reducing the structure response under the harmonic and the seismic load. Furthermore, the proposed technique is robust to the variation of the amplitude of the excitation load.

Acknowledgments: Financial support by Hibah Bersaing research fund from The Ministry of Research and Technology and Higher Education of Indonesia with contract number 55/UN.16/HB/LPPM/2016 is gratefully acknowledged.

\section{References and Notes}

1. J.C. Ramallo, and A.M. Johnson, J. Eng. Mech. 128, 10 (2002).

2. E.G. Thermou, S. Pantazopoulou, and A.S. Elnashai, J. Earthquake Eng. 14,5 (2010).

3. H. Kim, and H. Adeli, J. Struc. Eng. 131, 12 (2015).

4. F. Ziegler, and AK. Amiri, Asian J. Civil Eng. 14,1 (2013).

5. O. Altay, C. Butenweg, S. Klinkel, and F. Taddei, Proceeding of the $9^{\text {th }}$ International Conference on Structural Dynamics(EURODYN) (2014).

6. J.P. Den Hartog. Mechanical Vibrations, Fourth Ed. McGraw-Hill Book Company. New York (1956).

7. L. Zuo, and S.A. Nayfeh, J. Sound and Vib. 272 (2004).

8. L. Son, M. Bur, and M. Rusli, Struct. Eng. and Mech. 57,1(2016).

9. L. Son, M. Bur, and M. Rusli, App. Mech. and Mat. 836(2016).

10. J.K. Yu, T. Wakahara, and D.A. Reed, Earthquake Eng. Struct. Dyn 28 (1999).

11. Y. Arfiadi, and Hadi M.N.S. Int. J. Optim. Civil Eng. 1(2011).
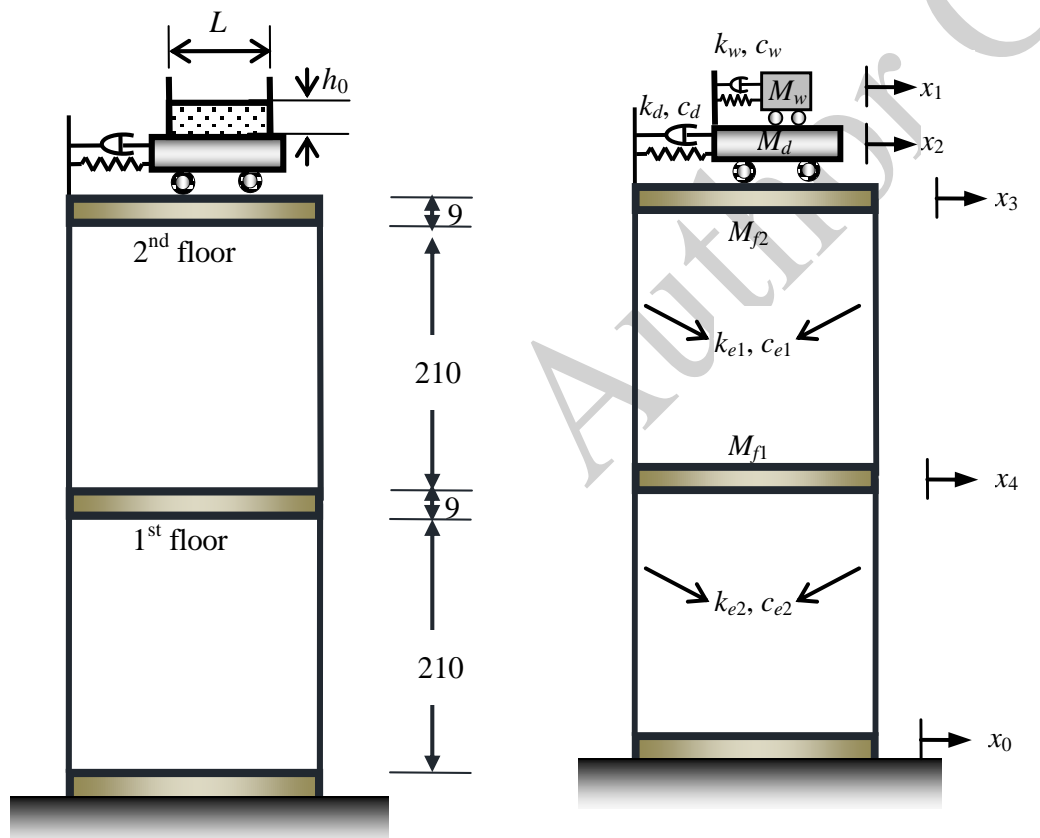

Fig. 1. Two-DOF shear structure with TMD and TLD. 


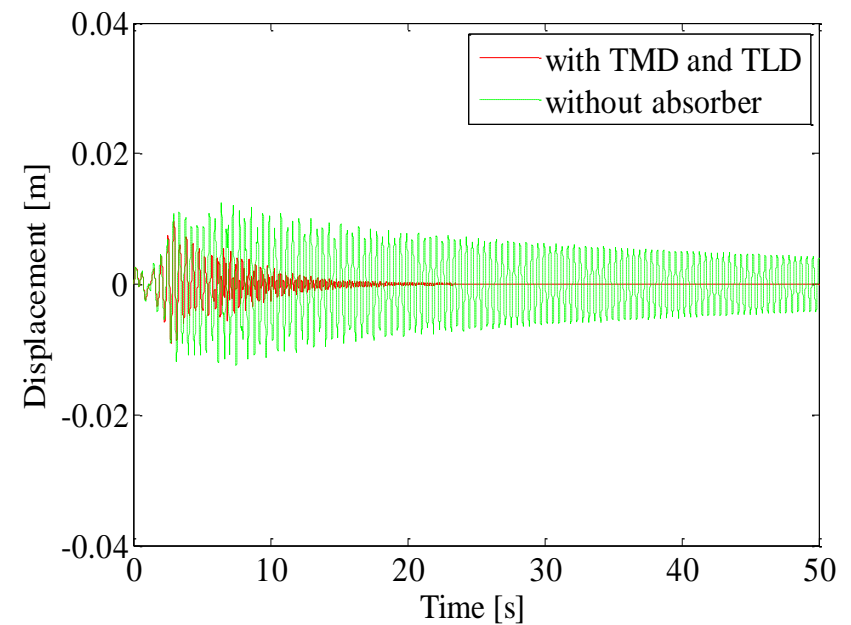

Fig. 2. Acceleration due to low level harmonic excitation.

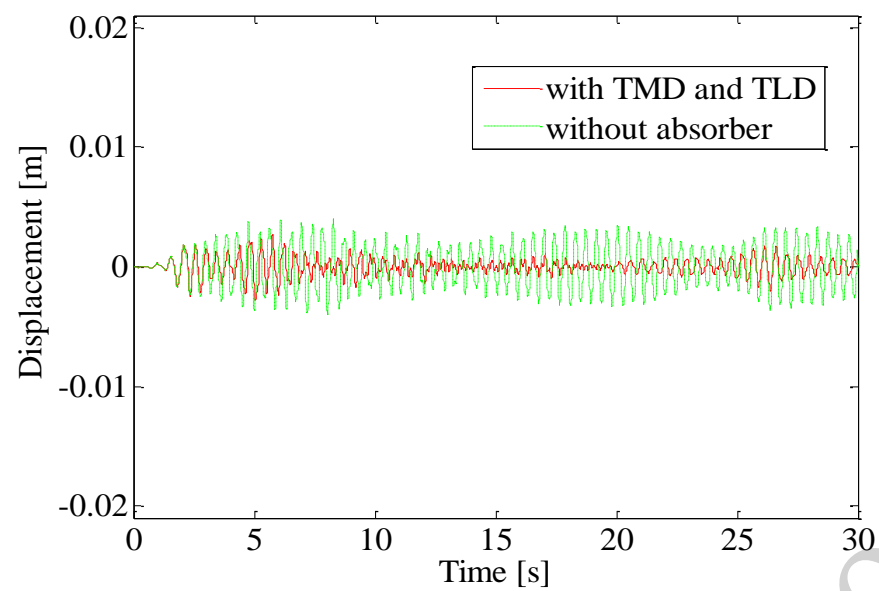

Fig. 4. Acceleration due to low level El-centro excitation.

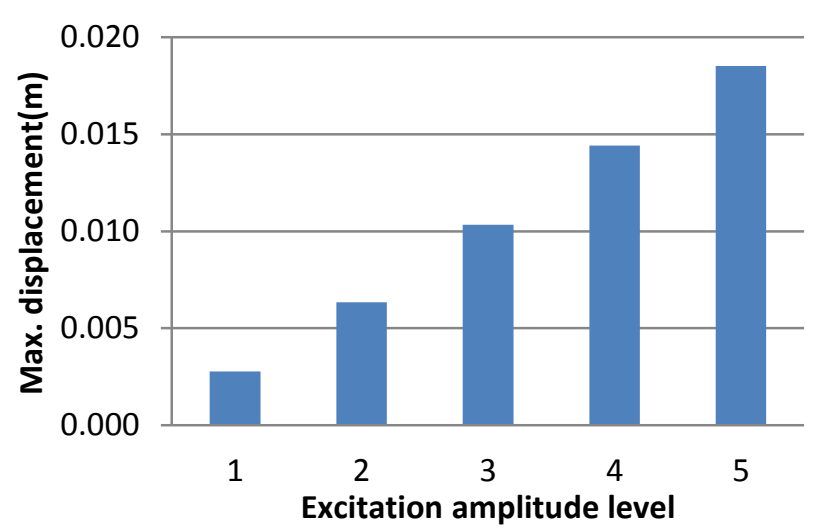

Fig. 6. Comparison of maximum amplitude

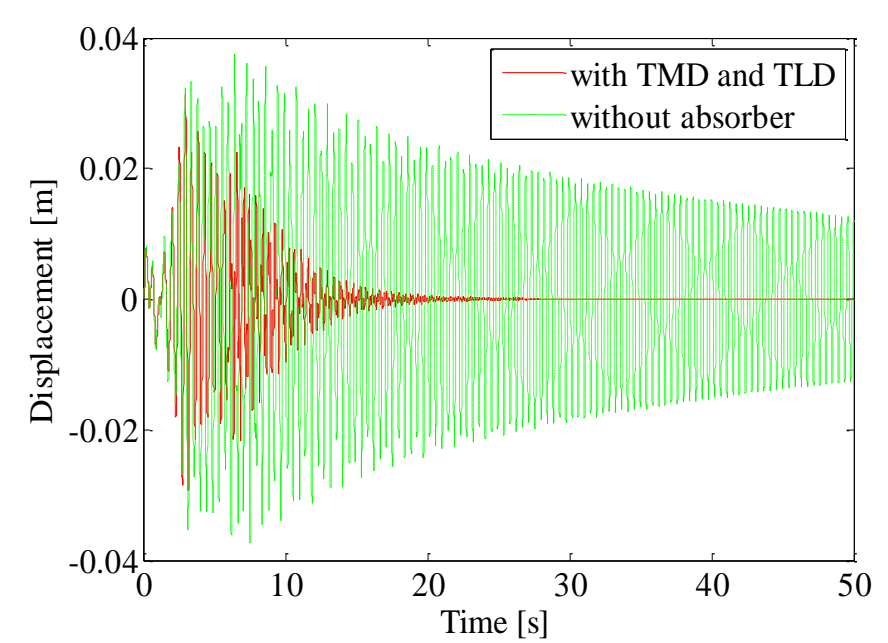

Fig. 3. Acceleration due to high level harmonic excitation.

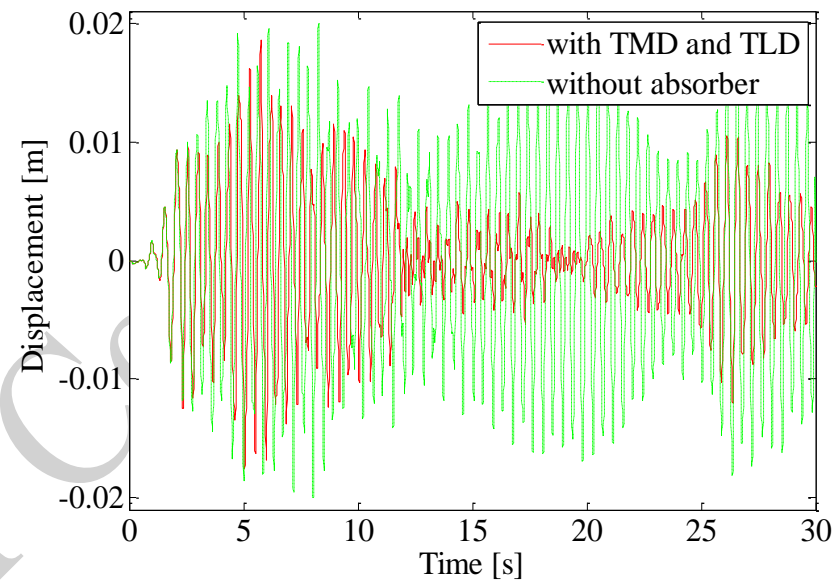

Fig.5. Acceleration due to high level El-centro excitation.

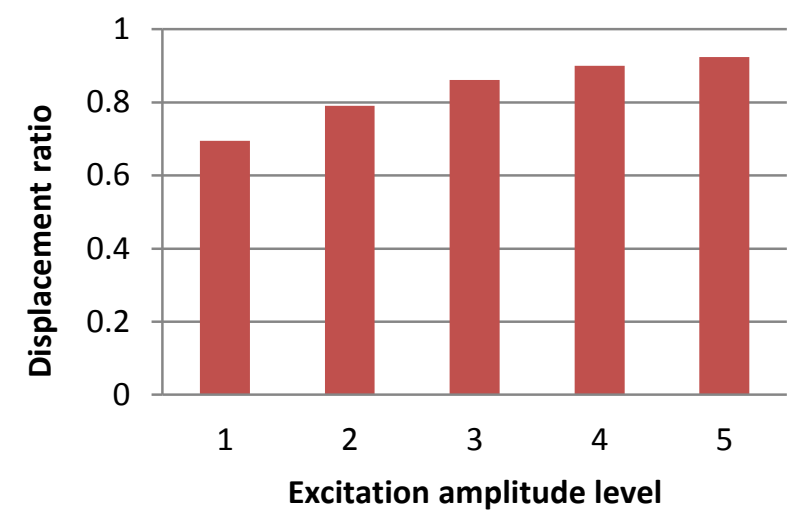

Fig.7. Displacement ratio

Table 1: Simulation parameters

\begin{tabular}{|l|l|l|}
\hline No & Parameters & Value \\
\hline 1 & Mass of the $1^{\text {st }}$ floor $\left(M_{f 1}\right)$ & $3.035 \mathrm{~kg}$ \\
\hline 2 & Mass of the $2^{\text {nd }}$ floor $\left(M_{f 2}\right)$ & $3.010 \mathrm{~kg}$ \\
\hline 3 & Structural stiffness $\left(k_{e 1}, k_{e 2}\right)$ & $1.64 \times 10^{3} \mathrm{~N} / \mathrm{m}$ \\
\hline 4 & Structural damping coefficient $\left(c_{e 1}, c_{e 2}\right)$ & $0.3 \mathrm{Ns} / \mathrm{m}$ \\
\hline 5 & Water density $(r)$ & $1000 \mathrm{~g} / \mathrm{m}^{3}$ \\
\hline 6 & TMD mass $\left(M_{d}\right)$ & $0.1 \mathrm{~kg}$ \\
\hline 7 & Weighting factor $(w$ and $a)$ & 0.5 \\
\hline 8 & Constant of gravitation $(g)$ & $9.81 \mathrm{~m} / \mathrm{s}^{2}$ \\
\hline
\end{tabular}

